\title{
Sedentary behavior, exercise, and cancer development
}

\author{
Tatjana T. König, MD*, Oliver J. Muensterer, MD, PhD
}

According to the World Health Organization, the global decrease of physical activity is one of the leading causes of mortality worldwide, resulting not only in cardiopulmonary disease and obesity, but also in a predisposition for the development of cancer ${ }^{[1]}$. This article gives an up-date perspective on the impact of sedentary behavior and exercise on cancer development, course of treatment, as well as secondary prevention in cancer survivors.

Inactivity is estimated to contribute to almost $25 \%$ of the global colorectal and breast cancer burden ${ }^{[1]}$. Even though the correlation is clear in large epidemiological series, showing a risk reduction of up to $20 \%$ for certain kinds of tumors in physically active individuals ${ }^{[2]}$, the underlying molecular mechanisms are mostly unknown.

Since malignant tumors are biologically active and favor aerobic glycolysis, tumor metabolism may be affected by energyconsuming activities, ultimately leaving the tumor in a more vulnerable state ${ }^{[3]}$.

Generally, a high level of inflammation within the tumor is associated with a better prognosis ${ }^{[3]}$. In rodents, physical activity lead to an activation of inflammatory cells and their attachment to tumors, increased levels of proinflammatory cytokines, and activation of proinflammatory signaling pathways, driven by an adrenergic physical stress response and an increase in body temperature ${ }^{[3]}$.

Physical activity seems to be a protective factor against tumors linked to hormonal changes in women, such as cancer of the breast and endometrium ${ }^{[2]}$. This is most likely facilitated by decreasing estradiol levels and increasing sex hormone binding globulin, both in premenopausal as well as postmenopausal women ${ }^{[4]}$. Furthermore, there is a correlation between metabolic activity and the development of intestinal neoplasms, such as esophageal, gastric, and colorectal ${ }^{[2]}$. This association remained

Department of Pediatric Surgery, Universitätsmedizin of the Johannes Gutenberg University Mainz, Mainz, Germany

This manuscript has been peer reviewed.

Sponsorships or competing interests that may be relevant to content are disclosed at the end of this article.

*Corresponding author. Address: Department of Pediatric Surgery,

Universitätsmedizin Mainz, Langenbeckstrasse 1, Mainz 55131, Germany.

Tel.: + 49613117 7107; fax: + 496131176523 . E-mail address:

tatjana.koenig@unimedizin-mainz.de (T.T.König).

Copyright $\odot 2019$ The Authors. Published by Wolters Kluwer Health, Inc. on behalf of IJS Publishing Group Ltd. This is an open access article distributed under the

Creative Commons Attribution License 4.0 (CCBY), which permits unrestricted use, distribution, and reproduction in any medium, provided the original work is properly cited.

International Journal of Surgery Oncology (2019) 4:e78

Received 14 August 2019; Accepted 20 August 2019

Published online 18 October 2019

http://dx.doi.org/10.1097//J9.00000000000000078

statistically significant ${ }^{[2]}$, even after controlling for body mass index.

For certain tumors associated with smoking and alcohol consumption, physical inactivity was shown to be protective (lung, liver, kidney, bladder, head, and neck tumors $)^{[2]}$. Surprisingly, among the listed tumors, smoking remained a more prominent independent risk factor compared to exercise only for lung cancer in a multivariate analysis. Hence, physically active smokers still had a reduced risk for liver, bladder, or head, and neck tumors, compared with physically inactive smokers ${ }^{[2]}$. Physical activity on the other hand was associated with a higher risk for malignant melanoma (hazard ratio, 1:1.27) and prostate cancer (hazard ratio, 1:1.05) ${ }^{[2]}$. While the higher risk for malignant melanoma might be explained by a higher exposure to sunlight in active individual, the data regarding the effect on prostate cancer remains inconclusive ${ }^{[5]}$. Conflicting studies found that physical activity may even have a preventive effect toward prostate cancer in some cases ${ }^{[5]}$.

In conclusion, physical activity seems to inhibit cancer development and progression by a combination of metabolic, endocrine, immunological, and inflammatory modulation ${ }^{[2,3]}$.

Cancer diagnosis and therapy itself has negative impact on the patient's physical fitness. Contributing factors include tumorassociated catabolism, fatigue, chemotherapy side-effects, extensive and repetitive surgery, as well as mental impact. There is evidence that a combination of weight control and physical activity is effective to reduce tumor growth, delay metastatic progression and improve survival ${ }^{[3]}$. Good muscular fitness is also associated with lower mortality and decreased risk of complications, as well as better adaption to treatment ${ }^{[3]}$. Preclinical research has described a muscle-to-tumor interaction via myokines and signaling peptides released by the muscle during exercise ${ }^{[3]}$.

Regarding objective treatment outcomes, physically active patients report a higher overall health-related quality of life (HrQOL), lower fatigue and fewer side-effects of chemotherapy ${ }^{[3]}$. By increasing overall blood flow and body temperature, physical activity has been shown not only to attenuate the toxicity of chemotherapy agents, but to improve their potency ${ }^{[3]}$.

Many prospective studies suggest that "prehabilitation," defined as the implementation of exercise programs during neoadjuvant treatment, can improve postoperative outcome even more effectively than rehabilitation after major surgery ${ }^{[6,7]}$. This effect has been demonstrated for major thoracic surgery in patients with lung cancer ${ }^{[7]}$, as well as major abdominal surgery ${ }^{[8]}$. The interpretation of these studies is hampered by heterogenous study protocols, especially in terms of exercise programs (inspiratory muscle training, "functional" training, endurance, resistance, or both, varying intensity levels and exercise frequency) and diverse outcome measures [self-reported physical activity, walking distance, maximal oxygen uptake $\left(\mathrm{VO}_{2}\right.$ max), grip strength $]^{[6-8]}$. Even among patients with the same 
tumor type, the response to exercise may vary widely ${ }^{[3]}$. These factors limit drawing any general conclusions with regard to pretreatment conditioning at this time.

As multimodal therapies improve, the focus of care is shifting towards improving long-term quality of life of cancer survivors. Even after successful tumor treatment, patients may experience live-long impairment in exercise-tolerance, as well as cardiopulmonary and cognitive function. Persisting symptoms of fatigue $^{[9]}$, compromised physical function, psychological comorbidities or chronic pain create a high barrier for participation in physical activity. Extensive tumor operations, such as pelvic exenterations, have been associated with a significant decrease in postoperative physical activity ${ }^{[10]}$. On the other hand, exercise improves autonomy by maintaining a higher level of cardiopulmonary and muscular function, coping abilities, as well as objective quality of life of cancer survivors ${ }^{[11]}$. A more active life-style before surgery seems to correlate with higher postoperative physical activity as well ${ }^{[10]}$. In large cohorts, longer survival has been demonstrated in cancer patients who adhere to a healthy life-style (nonsmoking, exercise, normal body mass index, healthy eating, moderate alcohol intake $)^{[12]}$. Finally, in multivariate regression analysis, exercise was found to be an independent factor for reduced overall mortality ${ }^{[12]}$.

Exercise also changes the long-term outlook. In a study of over 15,000 adult childhood cancer survivors followed-up over 10 years, tumor progression, relapse, and mortality was reduced by $40 \%$ in physically active patients compared to those with sedentary lifestyles ${ }^{[13]}$. Also, there was a positive linear dose-effect correlation between intensity and duration of exercise ${ }^{[13]}$. In this study, the optimal exercise exposure for childhood cancer survivors was modeled at 15-18 Metabolic Equivalent of Task $(\mathrm{MET}) \times$ hours per week $^{[13]}$, corresponding to about 2 hours of weekly cycling at $25 \mathrm{~km} /$ $\mathrm{h}^{[14]}$. In order to promote a habit of regular exercise, a growing number of home-based or community-based programs for cancer survivors have been implemented and adjusted to the patients' needs.

In conclusion, there is clear epidemiological evidence that links a sedentary life-style to cancer. Cancer patients have a better quality of life if they are physically active during their treatment. So-called "prehabilitation" during neoadjuvant treatment can improve surgical outcome and maintain a higher level of physical function. The scientific evidence on exercise-related treatment outcomes in cancer is currently limited to small series with heterogenous study protocols and patient profiles. Home and community-based exercise programs achieve a higher long-term adherence to an active lifestyle that may translate into a longer and healthier remission. Ultimately, these interventions must be tailored to the patients' needs, abilities, and background.

\section{Ethical approval}

None.

\section{Sources of funding}

Departmental funds.

\section{Author contribution}

T.T.K.: compilation of the literature. T.T.K., O.J.M.: preparation of the manuscript.

\section{Conflict of interest disclosure}

The author declares that there is no financial conflict of interest with regard to the content of this report.

\section{Research registration unique identifying number (UIN)}

None.

\section{Guarantor}

None.

\section{References}

[1] WHO. Global Recommendations on Physical Activity for Health. Geneva: WHO Press; 2010.

[2] Moore SC, Lee IM, Weiderpass E, et al. Association of leisure-time physical activity with risk of 26 types of cancer in 1.44 million adults. JAMA Intern Med 2016;176:816-25.

[3] Hojman P, Gehl J, Christensen JF, et al. Molecular mechanisms linking exercise to cancer prevention and treatment. Cell Metab 2018;27:10-21.

[4] de Boer MC, Worner EA, Verlaan D, et al. The mechanisms and effects of physical activity on breast cancer. Clin Breast Cancer 2017;17:272-8.

[5] Shephard RJ. Physical activity and prostate cancer: an updated review. Sports Med (Auckland, NZ) 2017;47:1055-73.

[6] Faithfull S, Turner L, Poole K, et al. Prehabilitation for adults diagnosed with cancer: a systematic review of long-term physical function, nutrition and patient-reported outcomes. Eur J Cancer Care 2019;28:e13023.

[7] Rosero ID, Ramirez-Velez R, Lucia A, et al. Systematic review and metaanalysis of randomized, controlled trials on preoperative physical exercise interventions in patients with non-small-cell lung cancer. Cancers 2019;11:E944.

[8] Heger P, Probst P, Wiskemann J, et al. A Systematic Review and Metaanalysis of Physical Exercise Prehabilitation in Major Abdominal Surgery (PROSPERO 2017 CRD42017080366). J Gastrointest Surg 2019. Doi: 10.1007/s11605-019-04287.

[9] Berntsen S, Aaronson NK, Buffart L, et al. Design of a randomized controlled trial of physical training and cancer (Phys-Can)-the impact of exercise intensity on cancer related fatigue, quality of life and disease outcome. BMC Cancer 2017;17:218.

[10] Steffens D, Young JM, Solomon M, et al. Preliminary evidence for physical activity following pelvic exenteration: a pilot longitudinal cohort study. BMC Cancer 2019;19:661.

[11] McDonough MH, Beselt LJ, Daun JT, et al. The role of social support in physical activity for cancer survivors: a systematic review. Psychooncology 2019. Doi: $10.1002 /$ pon.5171.

[12] Karavasiloglou N, Pestoni G, Wanner M, et al. Healthy lifestyle is inversely associated with mortality in cancer survivors: results from the Third National Health and Nutrition Examination Survey (NHANES III). PloS One 2019;14:e0218048.

[13] Scott JM, Li N, Liu Q, et al. Association of exercise with mortality in adult survivors of childhood cancer. JAMA Oncol 2018;4:1352-8.

[14] Jette MSK, Blümchen G. Metabolic equivalents (METS) in exerceise testing, exercise prescription and evaluation of functional capacity. Clin Cardiol 1990;13:555-65. 